\title{
Identification of the mineralocorticoid receptor in human spermatozoa
}

\author{
CRISTINA FIORE ${ }^{1}$, DANIELE STICCHI ${ }^{2}$, DONATELLA PELLATI ${ }^{1}$, SANTE FORZAN ${ }^{1}$, \\ GUGLIELMO BONANNI $^{1}$, ALESSANDRO BERTOLDO ${ }^{1}$, MICHELE MASSIRONI ${ }^{1}$, \\ LORENZO CALò $^{2}$, AMBROGIO FASSINA $^{3}$, GIAN PAOLO ROSSI ${ }^{2}$ and DECIO ARMANINI ${ }^{1}$ \\ Departments of ${ }^{1}$ Medical and Surgical Sciences (Endocrinology), ${ }^{2}$ Clinical and Experimental Medicine \\ (Internal Medicine 4), and ${ }^{3}$ Oncology and Surgical Sciences, University of Padua, 35100 Padua, Italy
}

Received March 31, 2006; Accepted June 5, 2006

\begin{abstract}
Aldosterone seems to play a role in the regulation of the electrolyte content of sperm and in the motility of spermatozoa. The aim of the study was to evaluate the presence of the mineralocorticoid receptor (MR) in human ejaculated spermatozoa. We have assayed MR on spermatozoa of freshly ejaculated sperm from healthy donors. The identification of MR was made by using immunohistochemistry and immunofluorescence analyses, while MR mRNA expression was evaluated by real-time PCR assay. The immunohistochemical and immunofluorescence analyses showed positive staining both in the midpiece and in the tail of the spermatozoa. Relative quantification of MR by using real-time PCR shows that the mRNA expression of MR in spermatozoa is lower than in mononuclear leukocytes (positive controls). Sequencing showed complete identity between the sequence obtained from spermatozoa and the human MR cDNA sequence. Further studies should be performed in order to elucidate a possible physiological role of aldosterone in regulating electrolyte concentration, and the pro-oxidant effect of excess aldosterone in this new target tissue.
\end{abstract}

\section{Introduction}

Mineralocorticoid receptors (MR) are not only present in classical epithelial tissues, such as the kidney and salivary glands, but they have also been identified in non-epithelial tissues, including the cardiovascular system, where MR seem to carry out an important role in the pathophysiology of the process of fibrosis $(1,2)$. We have previously demonstrated the

Correspondence to: Dr Decio Armanini, Department of Medical and Surgical Sciences - Endocrinology, University of Padua, Via Ospedale 105, 35128 Padua, Italy

E-mail: decio.armanini@unipd.it

Key words: mineralocorticoid receptors, spermatozoa, immunostaining, real-time PCR, sequencing presence of MR also in mononuclear leukocytes (MNL), where aldosterone regulates the intracellular content of electrolytes and volume (3-5) and is able to induce oxidative stress when produced in excess. This effect is reversed by the addition of canrenone, the main metabolite of spironolactone (6).

The action of physiological corticosteroids at the gonadal level is modulated by the two isoforms of the enzyme 11ßhydroxysteroid dehydrogenase (11HSD), which perform different roles in a wide range of reproductive processes (7).

MR have been found in Leydig cells, where both type 1 (11HSD1) and type 2 (11HSD2) 11HSD play a protective role opposing the adverse effects of excess cortisol (8). Evidence suggests a role for aldosterone both in regulation of the electrolyte content of sperm and in the motility of spermatozoa (9). Previous studies have shown a cause effect relationship between mineralocorticoid and glucocorticoid dysfunction and infertility in patients with varicocele (10). Mineralocorticoid receptors are also located in the epididymis where aldosterone can modulate ion fluxes (11). Both inhibition and removal of aldosterone cause a significant reduction in intraluminal sperm concentration (12). Based on these observations the aim of our study was to evaluate the presence of MR in human spermatozoa.

\section{Materials and methods}

Subjects. All assays were performed with freshly ejaculated sperm from healthy donors (6 males; age range, 21-35 years), obtained by masturbation after 3 days of sexual abstinence. The volunteers were fully informed about the experiment and they gave their consent. The study was approved by the local ethics committee. Sperm samples with normal parameters of semen volume, sperm count, motility, vitality, and morphology, according to the WHO Laboratory Manual, were included in this study.

Spermatozoa preparation. After semen analysis, the ejaculated samples were divided into two aliquots. The first aliquot was used to separate spermatozoa with high motility after swimup procedure $\left(1 \mathrm{~h} / 37^{\circ} \mathrm{C}\right)$ in RPMI-1640 (Serva, Italy). The remaining part of the semen sample was overlaid on a discontinuous Percoll gradient $(47 / 90 \%)$ and centrifuged at 
Table I. Primer sequence and expected size of PCR products for MR.

\begin{tabular}{llcc}
\hline Gene & Primer sequence & Size (bp) & Temperature \\
\hline \multirow{2}{*}{ hMR } & F: 5'- GCTTTGATGGTAACTGTGAAGG-3' & 471 & $68^{\circ} \mathrm{C}$ \\
& R: 5'- TGTGTTGCCCTTCCACTGCT-3' & & \\
GAPDH & F: 5'-GGGAAGGTGAAGGTGGGAGTG-3' & 375 & $65^{\circ} \mathrm{C}$ \\
\hline
\end{tabular}

$450 \mathrm{x} \mathrm{g}$ for $30 \mathrm{~min}$ at room temperature. The seminal plasma was discarded and the spermatozoa from the $47 \%$ Percoll interface and from the bottom pellet (90\% Percoll) were collected. The cells were washed and analyzed with regard to their concentration, motility, viability and morphology.

Immunohistochemistry and immunofluorescence assay. Isolated spermatozoa were resuspended in PBS medium at a concentration of $10^{6} / \mathrm{ml}$, and approximately $10 \mu 1$ was smeared on clean, grease-free slides, air-dried, and fixed in chilled acetone for $30 \mathrm{~min}$. Both analyses for MR were performed by using a mouse monoclonal antibody (H10E, Vinci-Biochem), directed at the aldosterone-binding site $(13,14)$, at a dilution of 1:200. For immunohistochemistry assay the antigenantibody complex was visualized with 3.3'-diaminobenzidine (DAB) solution (EnVision ${ }^{\mathrm{TM}}$, DakoCytomation, UK). The slides were counterstained with $1 \%$ hematoxylin, dehydrated in ascending grades of alcohol, kept in xylene, then mounted and assessed under light microscopy. For immunofluorescence assay we used goat anti-mouse-IgG-rhodamine red and counterstaining with 4'-6-diamidino-2-phenylindole (DAPI). The slides were examined under a fluorescence miscroscope.

MNL were used as positive controls, while the samples incubated with secondary antibody alone were used as negative controls. Pre-incubation with an excess of aldosterone completely abolished immunostaining, which was unaffected by excess RU-486, a steroid antagonist that does not bind to MR.

RNA isolation and real-time PCR assay. Total RNA was isolated from human ejaculated spermatozoa using the commercial product RNAzol B (Celbio) according to the manufacturer's instructions. The concentration of extracted RNA was determined by spectrophotometry at $260 \mathrm{~nm}$ absorbance. For the RT-PCR, 20 pmol of each specific primer were used (forward primer MR from +1649nt to +1670nt) and the reverse primer MR was constructed between exon 3 and exon 4 (from +2102nt to +2122nt; AC M16801, Genebank), to avoid the contamination of genomic DNA (Table I). Complementary DNA was synthesized from $0.5 \mu \mathrm{g}$ of RNA, using an iScript cDNA synthesis kit (BioRad).

Hot-start real-time PCR was performed using the Kit LightCycler Fast Start Master Plus SYBR-Green I (Roche), with human lymphocytes as positive control for MR, and for the relative quantification we used GAPDH housekeeping (Table I). Both the amplifications and the product revelation steps were performed in a glass capillary using a LightCycler (Roche). Sequencing was performed on double-stranded
DNA using the ABI PRISM dye terminator cycle sequencing core kit (Applied Biosystems, Monza, Italy). Electrophoresis of sequencing reactions was completed in an ABI PRISM automated sequencer, model 377 , version 2.1.1. The homology searches were carried out using the Basic Blast program, version 2.0, at http://www.ncbi.nlm.nih.gov/blast.

\section{Results}

The immunohistochemical analysis showed a positive staining both in the midpiece and in the tail of the spermatozoa. A positive staining is also present in MNL (used as a positive control) subjected to the same procedure (Fig. 1A). Negative controls, where the primary antibody was omitted, revealed no staining (Fig. 1B). Positive staining for MR was also observed, by immunofluorescence, in the midpiece region and in the tail region of the spermatozoa, confirming the immunohistochemical analysis (Fig. 2A). No immunoreaction was detected in the negative controls, thus demonstrating the immunostaining specificity (Fig. 2B). Pre-incubation with an excess of aldosterone completely abolished immunostaining, which was unaffected by excess RU-486, a steroid antagonist that does not bind to MR.

The MR mRNA expression, evaluated by RT-PCR analysis in different spermatozoa samples, is shown in Fig. 3. Relative quantification was performed in spermatozoa (Fig. 3a-g) and in MNL (Fig. 3h and i) normalizing for GAPDH expression. The results show that the number of threshold cycle $(\mathrm{Ct})$ for the MR mRNA expression was lower in MNL than spermatozoa thus demonstrating a higher expression in the first tissue.

Sequencing showed complete identity between the sequence obtained from spermatozoa and human mineralocorticoid receptor cDNA sequence (AC M16801).

\section{Discussion}

We have previously characterized MR in MNL (3); in these cells aldosterone not only regulates intracellular electrolytes (4) and volume (5), but excess aldosterone can also enhance the protein expression of oxidative stress-related genes, such as PAI-1 and p22 $2^{\text {phox }}(6)$. We have now demonstrated by immunohistochemistry, immunofluorescence and real-time PCR, the presence of MR in human spermatozoa. We used a monoclonal auto-anti-idiotypic antibody that recognizes the binding site of the receptor. The receptor-like specificity found in the competition experiment supports the specific detection of MR, consistently with the results obtained by Lombes et al in kidney (14). 
A

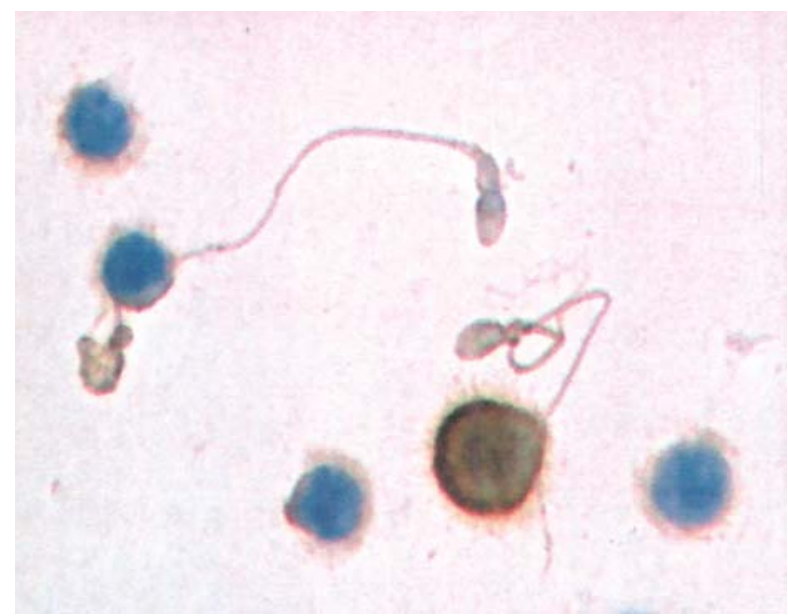

B

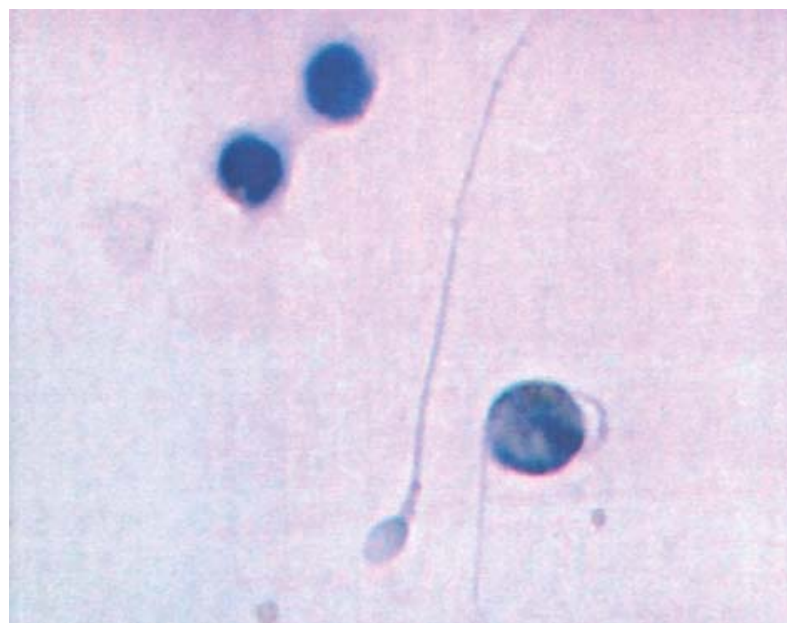

Figure 1. Immunohistochemical staining of MR in MNL and spermatozoa: (A) positive staining: spermatozoa and MNL appear brown as a result of diaminobenzidine colorimetric reaction; (B) negative control, sample without primary antibody.

MR in spermatozoa are less expressed than in MNL, but we hypothesize that aldosterone could play a physiological role in spermatozoa as previously found in other components of the reproductive system. The localization of MR in spermatozoa, in epididymal and Leydig cells contributes to the normal function of these components in relation to the reproductive potency. It is worth noting that previous studies have demonstrated the presence of 11HSD1 in human spermatozoa (8). 11HSD complex plays a crucial role in regulating the reproductive function both in females and in males, where it can modulate the activity of androgens via protection from the adverse effects of glucocorticoids $(7,8)$. No data are reported regarding 11HSD2. In the steroidogenic cells of testis the activity of NADPH-dependent cytochrome P450 enzymes favours the oxidation of NADPH to NADP, thus promoting the oxidative activity of 11HSD1 $(7,8)$. Such an effect could also be active in spermatozoa, allowing aldosterone to bind to its receptors.

In conclusion, we suggest that aldosterone excess could be involved in spermatozoa motility not only by deregulating the electrolyte composition of spermatic fluid and spermatozoa, but also by producing inflammatory effects, as demonstrated for MNL (6). Several studies have investigated or are in progress to evaluate the relationship between oxidative stress
A

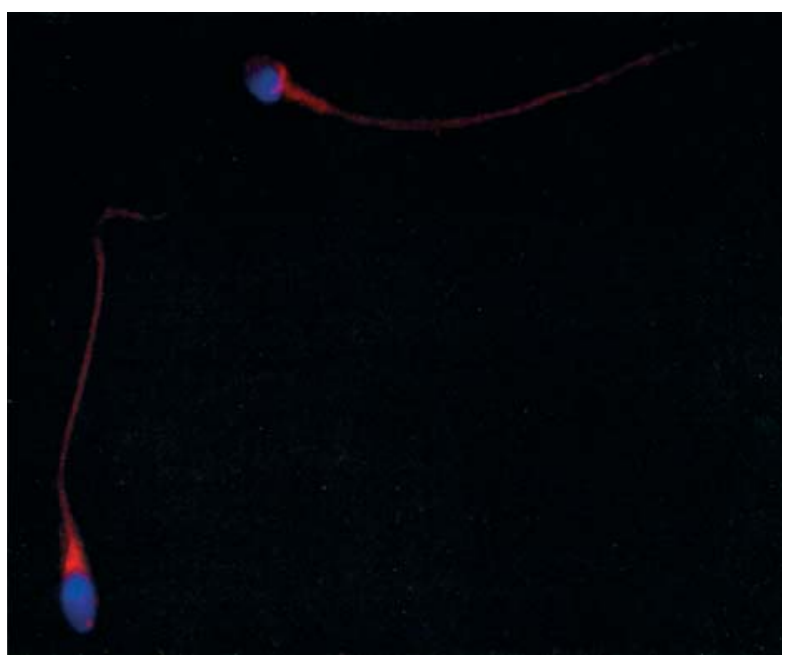

$\mathbf{B}$

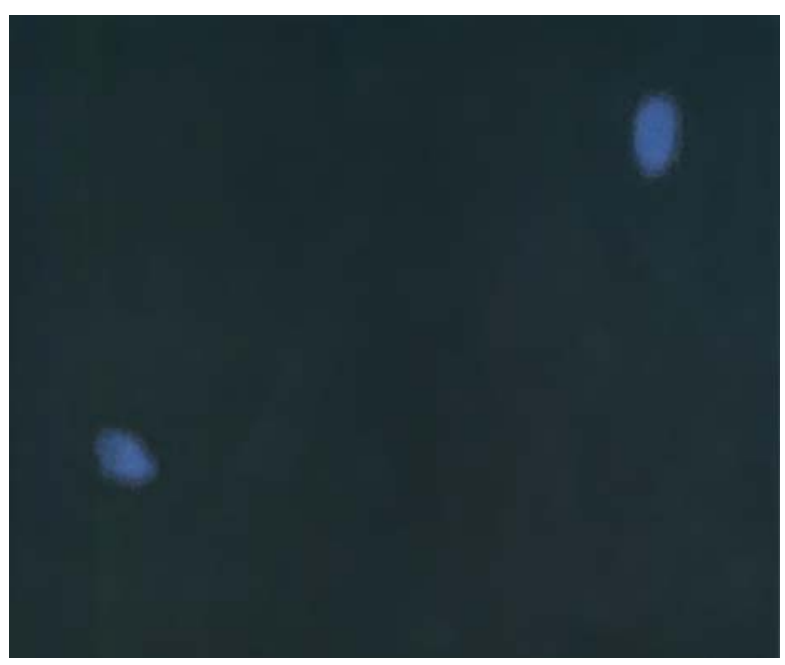

Figure 2. Immunofluorescence staining of MR in spermatozoa: (A) positive staining after incubation with H10E primary antibody and goat anti-mouseIgG-rhodamine red and DAPI; (B) negative control, sample without primary antibody.

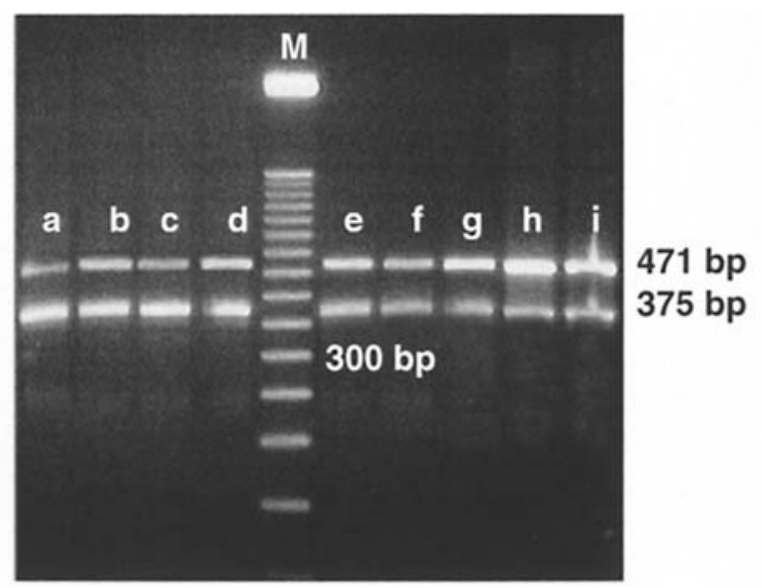

Figure 3. RT-PCR analysis of MR (471 bp) in seven different spermatozoa samples (a-g) and two different samples of MNL (h and i); RT-PCR analysis of GAPDH (375 bp) in the same spermatozoa samples (a-g) and MNL (h and i). Marker 50-bp step ladder (M).

and spermatozoa dysfunction, such as reduced motility and ability to fertilize the oocyte through damage to the nuclear 
DNA of these cells (15-17). Further studies need to be performed in order to clarify the role of aldosterone and MR in this target tissue and to confirm our hypotheses.

\section{Aknowledgements}

This work was partially supported by a research grant of the Italian Society of Hypertension.

\section{References}

1. Young MJ and Funder JW: Mineralcorticoid receptors and pathophysiological roles for aldosterone in the cardiovascular system. J Hypertens 20: 1465-1468, 2002.

2. Weber KT: From inflammation to fibrosis: a stiff stretch of highway. Hypertension 43: 716-719, 2004.

3. Armanini D, Strasser T and Weber PC: Characterization of aldosterone binding sites in circulating human mononuclear leucocytes. Am J Physiol 248: E388-E390, 1985.

4. Wehling M, Armanini D, Strasser T and Weber PC: Effect of aldosterone on sodium and potassium concentration in human mononuclear leucocytes. Am J Physiol 252: E505-E508, 1987.

5. Wehling M, Kuhls S and Armanini D: Volume regulation of human lymphocytes by aldosterone in isotonic media. Am J Physiol 257: E170-E174, 1989.

6. Calo LA, Zaghetto F, Pagnin E, Davis PA, De Mozzi P, Sartorato P, Martire G, Fiore C and Armanini D: Effect of aldosterone and glycyrrhetinic acid on the protein expression of PAI-1 and p22(phox) in human mononuclear leukocytes. J Clin Endocrinol Metab 89: 1973-1976, 2004.

7. Michael AE, Thurston LM and Rae MT: Glucocorticoid metabolism and reproduction: a tale of two enzymes. Reproduction 126: 425-441, 2003.
8. Nacharaju VL, Munevyirci-Delale O and Khan N: Presence of 11 beta-hydroxysteroid dehydrogenase in human semen: evidence of correlation with semen characteristics. Steroids 62: 311-314, 1997.

9. Rossato M, Balercia G, Lucarelli G, Foresta C and Mantero F: Role of seminal osmolarity in the reduction of human sperm motility. Int J Androl 25: 230-235, 2002.

10. Mazo EB, Koryakin MV, Kudryavtsev JuV, Evseev LP and Akopyan AS: The role of impairment of adrenal mineraloglucocorticoid function in the development of infertility in varicocele patients. Int Urol Nephrol 21: 403-416, 1989.

11. Turner TT and Cesarini DM: The ability of the rat epididymis to concentrate spermatozoa: Responsiveness to aldosterone. J Androl 4: 197-202, 1983

12. Wong PY and Lee WM: Effects of spironolactone (aldosterone antagonist) on electrolyte and water content of the cauda epididymis and fertility of male rats. Biol Reprod 27: 771-777, 1982.

13. Lombes M, Edelman IS and Erlanger BF: Internal image properties of a monoclonal auto-anti-idiotypic antibody and its binding to aldosterone receptors. J Biol Chem 264: 2528-2536, 1989.

14. Lombes M, Farman N and Oblin ME: Immunohistochemical localization of renal mineralocorticoid receptor by using an antiidiotypic antibody that is an internal image of aldosterone. Proc Natl Acad Sci 87: 1086-1088, 1990.

15. Aitken RJ and Baker MA: Oxidative stress and male reproductive biology. Reprod Fertil Dev 16: 581-588, 2004.

16. De Lamirande E and Gagnon C: Impact of reactive oxygen species on spermatozoa: a balancing act between beneficial and detrimental effects. Hum Reprod 10: 15-21, 1995.

17. Sharma RK and Agarwal A: Role of reactive oxygen species in male infertility. Urology 48: 835-850, 1996. 